Research Article

\title{
Improved Regularization Method for Backward Cauchy Problems Associated with Continuous Spectrum Operator
}

\author{
Salah Djezzar and Nihed Teniou \\ Laboratoire Equations Différentielles, Département de Mathématiques, Faculté des Sciences Exactes, \\ Université Mentouri Constantine, Constantine 25000, Algeria \\ Correspondence should be addressed to Salah Djezzar, salah_djezzar@yahoo.fr
}

Received 29 May 2011; Revised 17 August 2011; Accepted 26 September 2011

Academic Editor: Alberto Cabada

Copyright (C) 2011 S. Djezzar and N. Teniou. This is an open access article distributed under the Creative Commons Attribution License, which permits unrestricted use, distribution, and reproduction in any medium, provided the original work is properly cited.

\begin{abstract}
We consider in this paper an abstract parabolic backward Cauchy problem associated with an unbounded linear operator in a Hilbert space $H$, where the coefficient operator in the equation is an unbounded self-adjoint positive operator which has a continuous spectrum and the data is given at the final time $t=T$ and a solution for $0 \leq t<T$ is sought. It is well known that this problem is illposed in the sense that the solution (if it exists) does not depend continuously on the given data. The method of regularization used here consists of perturbing both the equation and the final condition to obtain an approximate nonlocal problem depending on two small parameters. We give some estimates for the solution of the regularized problem, and we also show that the modified problem is stable and its solution is an approximation of the exact solution of the original problem. Finally, some other convergence results including some explicit convergence rates are also provided.
\end{abstract}

\section{Introduction}

Let $A$ be a positive (we suppose that $A \geq \eta>0$ ), self-adjoint unbounded linear operator which has a continuous spectrum on a Hilbert space $H$ such that $-A$ generates a contraction $\mathrm{C}_{0}$-semigroup on $H$. Let $T$ be a positive real number. We consider the final value problem (FVP) of finding $u:[0, T] \rightarrow H$ such that

$$
\begin{gathered}
u^{\prime}(t)+A u(t)=0, \quad 0 \leq t<T, \\
u(T)=\phi,
\end{gathered}
$$


for some prescribed final value $\phi$ in $H$. Such problems are not well posed; that is, even if a unique solution exists on $[0, T]$, it need not depend continuously on the finial value $\phi$.

This type of problems, in the case where $A$ has a discrete spectrum, has been considered by many authors using different approaches. Such authors as Lattès and Lions [1], Lavrentiev [2], Miller [3], Payne [4], and Showalter [5] have approximated the final value problem (FVP) (1.1), (1.2) by perturbing the operator $A$.

A similar problem is treated in a different way; see [6-8]. By perturbing the final value condition, they approximated the problem (1.1), (1.2) with

$$
\begin{gathered}
u^{\prime}(t)+A u(t)=0, \quad 0 \leq t<T . \\
\alpha u(0)+u(T)=\phi .
\end{gathered}
$$

A similar approach known as the method of auxiliary boundary conditions was given in [9-11]. Also, the nonstandard conditions of the form (1.3) for parabolic equations have been considered in some recent papers $[12,13]$. For further results related to these type of problems, we can also see $[14,15]$. It is also worth reading the recent paper by Campbell Hetrick and Hunhes [16] dealing with inhomogeneous ill-posed problems in Banach space. We also mention the very recent papers by Tuan [17] and Tuan et al. [18] which deal with similar ill-posed problems using different approaches. For some comments on the results presented in paper [17] using a different regularization approach (the truncation regularization method), see Remark 3.6 at the end of this paper.

In this paper, we perturb both (1.1) and the final condition (1.2) to form an approximate nonlocal problem depending on two small parameters $\alpha$ and $\beta$, with boundary condition containing a derivative of the same order than the equation as follows:

$$
\begin{gathered}
v_{\sigma}^{\prime}(t)+A_{\alpha} v_{\sigma}(t)=0, \quad 0 \leq t<T, \\
v_{\sigma}(T)+\beta\left(v_{\sigma}(0)-v_{\sigma}^{\prime}(0)\right)=\phi,
\end{gathered}
$$

where the operator $A$ is replaced by the operator $A_{\alpha}=A(I+\alpha A)^{-1}$ and $u(T)=\phi$ by $u_{\sigma}(T)+$ $\beta\left(u_{\sigma}(0)-u_{\sigma}^{\prime}(0)\right)=\phi$, and $\sigma=(\alpha, \beta)$, where $\alpha>0, \beta>0$.

We show that the approximate problems are well posed and that their solutions $v_{\sigma}$ converge if and only if the original problem has a classical solution. We also show that this method gives a better approximation than many other quasireversibility and quasiboundary type methods, for example, [1, 6, 7, 19-21]. Finally, we obtain several other results, including some explicit convergence rates.

Throughout this paper, we will denote by $H$ a Hilbert space, $\left\{E_{\lambda}, \lambda \geq \eta>0\right\}$ the resolution of the identity associated with the positive unbounded self-adjoint operator $A$. So the spectral representation of $C_{0}$ semigroup $S(t)=e^{-t A}$ (resp., $A$ ) is given by $S(t)=e^{-t A}=$ $\int_{\eta}^{+\infty} e^{-t \lambda} d E_{\curlywedge}$ (resp., $A=\int_{\eta}^{+\infty} \lambda d E_{\curlywedge}$ ), and so for all $u \in D(A), A u=\int_{\eta}^{+\infty} \lambda d E_{\curlywedge} u$, and this is characterized by

$$
u \in D(A) \quad \text { iff }\|A u\|^{2}=\int_{\eta}^{+\infty} \lambda^{2} d\left\|E_{\lambda} u\right\|^{2}<\infty
$$


Also, throughout this work, we mean by a solution of problem (1.1), (1.2) on the interval $[0, T]$ a function $u \in C([0, T] ; H) \cap C^{1}(] 0, T[; H)$ such that, for all $\left.t \in\right] 0, T[, u(t) \in D(A)$ and (1.1), (1.2) hold. A useful characterization of the admissible set for which problem (1.1), (1.2) has a solution is as follows: Problem (1.1), (1.2) has a solution $u$ if and only if $\int_{\eta}^{+\infty} e^{2 \lambda T} d\left\|E_{\lambda} \phi\right\|^{2}<\infty$, and this unique solution is represented by $u(t)=\int_{\eta}^{+\infty} e^{(T-t) \lambda} d E_{\lambda} \phi<\infty$ (see [19], Lemma 1]).

\section{The Approximate Problem}

We approximate the final value problem (1.1), (1.2), by the following perturbed problem:

$$
\begin{gathered}
v_{\sigma}^{\prime}(t)+A_{\alpha} v_{\sigma}(t)=0, \quad 0 \leqslant t<T, \\
v_{\sigma}(T)+\beta\left(v_{\sigma}(0)-v_{\sigma}^{\prime}(0)\right)=\phi,
\end{gathered}
$$

where $A$ is as above and $A_{\alpha}$ is the Yosida approximation of operator $A$, and $\sigma=(\alpha, \beta)$ where $\alpha>0, \beta>0$.

Definition 2.1. Define the function

$$
v_{\sigma}(t)=S_{\alpha}(t)\left(\beta\left(I+A_{\alpha}\right)+S_{\alpha}(T)\right)^{-1} \phi,
$$

for $\phi \in H, \sigma=(\alpha, \beta)$ and $\alpha>0, \beta>0, t \in[0, T]$, where $S_{\alpha}(t)$ is the semigroup generated by $-A_{\alpha}$.

Now, we give the following theorem where the proof is based on the semigroups theory [22].

Theorem 2.2. The function $v_{\sigma}(t)$ is the unique solution of the perturbed problem (2.1), and it depends continuously on $\phi$.

Proof. We consider the following classical Cauchy problem:

$$
\begin{aligned}
& v_{\sigma}^{\prime}(t)+A_{\alpha} v_{\sigma}(t)=0, \quad 0<t<T, \\
& v_{\sigma}(0)=\left(\beta\left(I+A_{\alpha}\right)+S_{\alpha}(T)\right)^{-1} \phi .
\end{aligned}
$$

It is clear that $v_{\sigma}(t)$ is the unique solution and

$$
\begin{aligned}
v_{\sigma}(T)+\beta\left(v_{\sigma}(0)-v_{\sigma}^{\prime}(0)\right)= & S_{\alpha}(T)\left(\beta\left(I+A_{\alpha}\right)+S_{\alpha}(T)\right)^{-1} \phi \\
& +\beta\left(I+A_{\alpha}\right)\left(\beta\left(I+A_{\alpha}\right)+S_{\alpha}(T)\right)^{-1} \phi \\
= & \left(\beta\left(I+A_{\alpha}\right)+S_{\alpha}(T)\right)\left(\beta\left(I+A_{\alpha}\right)+S_{\alpha}(T)\right)^{-1} \phi=\phi .
\end{aligned}
$$


The continuous dependence of $v_{\sigma}$ on $\phi$ is obtained by showing that

$$
\begin{aligned}
\left\|v_{\sigma}(t)\right\| & =\left\|S_{\alpha}(t)\left(\beta\left(I+A_{\alpha}\right)+S_{\alpha}(T)\right)^{-1} \phi\right\| \\
& \leqslant\left\|\left(\beta\left(I+A_{\alpha}\right)+S_{\alpha}(T)\right)^{-1} \phi\right\| \\
& \leqslant \frac{1}{\beta+(\beta \eta /(1+\alpha \eta))+e^{-T / \alpha}}\|\phi\| .
\end{aligned}
$$

Now, we consider the following problem:

$$
\begin{gathered}
u_{\sigma}^{\prime}(t)+A u_{\sigma}(t)=0, \\
u_{\sigma}(0)=\phi_{\sigma}=\left(\beta\left(I+A_{\alpha}\right)+S_{\alpha}(T)\right)^{-1} \phi .
\end{gathered}
$$

Theorem 2.3. The problem (2.6) is well posed, and its solution is given by

$$
u_{\sigma}(t)=S(t)\left(\beta\left(I+A_{\alpha}\right)+S_{\alpha}(T)\right)^{-1} \phi,
$$

furthermore,

$$
\left\|u_{\sigma}(t)\right\| \leqslant \frac{1}{\left(\beta(1+(\eta /(1+\alpha \eta)))+e^{-T / \alpha}\right)^{(T-t) / T}}\|\phi\| .
$$

Proof. Since

$$
\begin{aligned}
\left\|u_{\sigma}(t)\right\|^{2} & =\int_{\eta}^{+\infty} \frac{e^{-2 \lambda T}}{\left(\beta(1+(\lambda /(1+\alpha \lambda)))+e^{-(\lambda T) /(1+\alpha \lambda)}\right)^{2}} d\left\|E_{\curlywedge} \phi\right\|^{2} \\
& \leq \int_{\eta}^{+\infty}\left[\frac{1}{\left(\beta(1+(\eta /(1+\alpha \eta)))+e^{-T / \alpha}\right)^{2}}\right]^{T-t / T} d\left\|E_{\curlywedge} \phi\right\|^{2},
\end{aligned}
$$

then,

$$
\left\|u_{\sigma}(t)\right\| \leqslant\left[\frac{1}{\left(\beta(1+(\eta /(1+\alpha \eta)))+e^{-T / \alpha}\right)}\right]^{T-t / T}\|\phi\| .
$$

Now we give some convergence results.

\section{The Convergence Results}

Theorem 3.1. For all $\phi \in H,\left\|u_{\sigma}(T)-\phi\right\| \rightarrow 0$, as $|\sigma| \rightarrow 0$. 
Proof. We have

$$
\begin{aligned}
\left\|u_{\sigma}(T)-\phi\right\|^{2} & =\int_{\eta}^{+\infty}\left(\frac{e^{-\lambda T}}{\beta(1+(\lambda /(1+\alpha \lambda)))+e^{-(\lambda T) /(1+\alpha \lambda)}}-1\right)^{2} d\left\|E_{\lambda} \phi\right\|^{2} \\
& =\int_{\eta}^{+\infty}\left(\frac{\beta(1+(\lambda /(1+\alpha \lambda)))}{\beta(1+(\lambda /(1+\alpha \lambda)))+e^{-(\lambda T /(1+\alpha \lambda))}}+\frac{e^{-(\lambda T /(1+\alpha \lambda))}-e^{-\lambda T}}{\beta(1+(\lambda /(1+\alpha \lambda)))+e^{-(\lambda T /(1+\alpha \lambda))}}\right)^{2} d\left\|E_{\lambda} \phi\right\|^{2} .
\end{aligned}
$$

Putting

$$
\begin{aligned}
& M_{\sigma}(\lambda)=\frac{\beta(1+(\lambda /(1+\alpha \lambda)))}{\beta(1+(\lambda /(1+\alpha \lambda)))+e^{-(\lambda T /(1+\alpha \lambda))}} \\
& N_{\sigma}(\lambda)=\frac{e^{-(\lambda T /(1+\alpha \lambda))}-e^{-\lambda T}}{\beta(1+(\lambda /(1+\alpha \lambda)))+e^{-(\lambda T /(1+\alpha \lambda))}},
\end{aligned}
$$

and if we put

$$
P_{\sigma}(\lambda)=M_{\sigma}(\lambda)+N_{\sigma}(\lambda)
$$

then we get

$$
\begin{aligned}
\left\|u_{\sigma}(T)-\phi\right\|^{2} & =\int_{\eta}^{+\infty}\left(P_{\sigma}(\lambda)\right)^{2} d\left\|E_{\lambda} \phi\right\|^{2} \\
& \leqslant 2 \int_{\eta}^{+\infty}\left(M_{\sigma}^{2}(\lambda)+N_{\sigma}^{2}(\lambda)\right) d\left\|E_{\curlywedge} \phi\right\|^{2}
\end{aligned}
$$

It is clear that, for all $\varepsilon>0$, there exists $k \in \mathbb{N}^{*} / \int_{k}^{+\infty} d\left\|E_{\lambda} \phi\right\|^{2}<\varepsilon / 8$ and $M_{\sigma}(\lambda) \leq 1, N_{\sigma}(\lambda) \leqslant 1$. If we put

$$
\begin{gathered}
I_{\sigma}=\int_{\eta}^{k} M_{\sigma}^{2}(\lambda) d\left\|E_{\lambda} \phi\right\|^{2}+\int_{k}^{+\infty} M_{\sigma}^{2}(\lambda) d\left\|E_{\lambda} \phi\right\|^{2}, \\
J_{\sigma}=\int_{\eta}^{k} N_{\sigma}^{2}(\lambda) d\left\|E_{\lambda} \phi\right\|^{2}+\int_{k}^{+\infty} N_{\sigma}^{2}(\lambda) d\left\|E_{\lambda} \phi\right\|^{2} .
\end{gathered}
$$

then we have

$$
\begin{gathered}
I_{\sigma} \leqslant \frac{\varepsilon}{8}+\beta^{2}\left(1+\frac{1}{\alpha}\right)^{2} e^{2 k T}\|\phi\|^{2}, \\
J_{\sigma} \leqslant \frac{\varepsilon}{8}+\alpha^{2} T^{2} k^{4}\|\phi\|^{2}
\end{gathered}
$$


Finally, choosing $\sigma$ such that $|\sigma|^{2}=\alpha^{2}+\beta^{2} \leqslant\left(1 /\|\phi\|^{2}\right)\left(1 / T^{2} k^{4}+1 / e^{2 k T}\right)(\varepsilon / 8)$, we obtain the following estimate

$$
\left\|u_{\sigma}(T)-\phi\right\|^{2} \leqslant\left(\frac{\epsilon}{2}+2\left(\beta^{2}\left(1+\frac{1}{\alpha}\right)^{2} e^{2 k T}+\alpha^{2} T^{2} k^{4}\right)\right)\|\phi\|^{2}
$$

which gives the desired result.

Let us denote by $C_{\theta}(A), \theta \geq 0$ the following set:

$$
C_{\theta}(A)=\left\{h \in H:\|h\|_{C_{\theta}}^{2}=\int_{\eta}^{+\infty} e^{2 T \theta \lambda} d\left\|E_{\lambda} \phi\right\|^{2}<+\infty\right\}
$$

It is clear that the following proprieties hold

$$
\begin{gathered}
C_{\theta_{1}}(A) \subseteq C_{\theta_{2}}(A), \quad \theta_{2} \geqslant \theta_{1}, \\
C_{\theta}(A) \subset H, \quad \theta>0 .
\end{gathered}
$$

Now, we give some convergence results with explicit convergence rates.

Theorem 3.2. If $\phi \in C_{\theta}(A)$, then one has

$$
\left\|u_{\sigma}(T)-\phi\right\|^{2} \leqslant 2\left(C_{1}^{2}(\theta) \frac{\beta^{2 \theta}}{\alpha^{2}}\left(\frac{\eta}{1+\alpha \eta}\right)^{2(\theta-1)}+C_{2}^{2}(\theta, T) \alpha^{2}\right)\|\phi\|_{C_{\theta^{\prime}}}^{2}
$$

for $0<\theta<1$ and

$$
\left\|u_{\sigma}(T)-\phi\right\|^{2} \leqslant 2\left(\frac{\beta^{2}}{\alpha^{2}}+C_{2}^{2}(\theta, T) \alpha^{2}\right)\|\phi\|_{C_{\theta^{\prime}}}^{2} \text { for } \theta \geqslant 1
$$

such that

$$
C_{1}(\theta)=(1-\theta)^{1-\theta} \theta^{\theta} \leqslant 1, \quad C_{2}(\theta, T)=\frac{1}{T \theta^{2}}
$$

Proof. Using the proof of the previous theorem, we have

$$
\begin{aligned}
\left\|u_{\sigma}(T)-\phi\right\|^{2} & =\int_{\eta}^{+\infty} P_{\sigma}^{2}(\lambda) d\left\|E_{\curlywedge} \phi\right\|^{2} \\
& =\int_{\eta}^{+\infty} P_{\sigma}^{2}(\lambda) e^{-2 T \theta \lambda} e^{2 T \theta \lambda} d\left\|E_{\lambda} \phi\right\|^{2} \\
& \leqslant 2\left(M_{\sigma, \theta, \infty}^{2}+N_{\sigma, \theta, \infty}^{2}\right)\|\varphi\|_{C_{\theta^{\prime}}}^{2}
\end{aligned}
$$


where

$$
M_{\sigma, \theta, \infty}=\sup _{l \geqslant \eta} M_{\sigma} e^{-T \theta \lambda}, \quad N_{\sigma, \theta, \infty}=\sup _{l \geqslant \eta} N_{\sigma} e^{-T \theta \lambda}
$$

If $0<\theta<1$, then we have

$$
M_{\sigma, \theta, \infty} \leq \frac{2}{\alpha} \beta^{\theta} C_{1}(\theta)
$$

And, for $\theta \geqslant 1$, we have

$$
M_{\sigma, \theta, \infty} \leq 2 \frac{\beta}{\alpha}
$$

We also have

$$
N_{\sigma, \theta, \infty} \leqslant \frac{4 \alpha}{T \theta^{2} e^{2}} \leqslant \frac{\alpha}{T \theta^{2}}, \quad \forall \theta>0
$$

Then, using the above estimates, we get the desired results.

Now, let $F$ be the function defined by

$$
\begin{aligned}
& F: \mathbb{R}^{+} \times \mathbb{R}^{+} \longrightarrow H \\
& \sigma=(\alpha, \beta) \longrightarrow F(\sigma)= \begin{cases}u_{\sigma}(0)=\phi_{\sigma}, & \sigma \neq(0,0) \\
u(0)=\phi_{0}, & \sigma=(0,0)\end{cases}
\end{aligned}
$$

Theorem 3.3. For all $\phi \in H$, the problem (1.1), (1.2) has a solution $u(t)$ if and only if the function $F$ is continious at $(0,0)$. Furthermore, $u_{\sigma}(t) \rightarrow u(t)$, as $|\sigma| \rightarrow 0$, uniformly in $t$.

Proof. We assume that $\lim _{|\sigma| \rightarrow 0} \phi_{\sigma}=\phi_{0}$ and $\left\|\phi_{0}\right\|<\infty$. Let $w(t)=S(t) \phi_{0}$. So, we have

$$
\left\|w(t)-u_{\sigma}(t)\right\| \leqslant\left\|\phi_{0}-\phi_{\sigma}\right\|
$$

Hence,

$$
\sup _{0 \leqslant \leqslant \leqslant T}\left\|w(t)-u_{\sigma}(t)\right\| \leqslant\left\|\phi_{0}-\phi_{\sigma}\right\| \underset{|\sigma| \rightarrow 0}{\longrightarrow} 0
$$

Since $\lim _{|\sigma| \rightarrow 0} u_{\sigma}(T)=\phi$ and $\lim _{|\sigma| \rightarrow 0} u_{\sigma}(T)=w(T)$ and so by the unicity of the limit, we obtain that $w(t)$ is a solution to problem (1.1), (1.2). 
Now, we suppose that $u(t)=\int_{\eta}^{+\infty} e^{(T-t) \lambda} d E_{\curlywedge} \phi$ is a solution to problem (1.1), (1.2). Since $u(0)=S(-T) \phi \in H$ (see [19], Lemma 1), then we have

$$
\|u(0)\|^{2}=\|\phi\|_{C_{1}}^{2}=\int_{\eta}^{+\infty} e^{2 T \lambda} d\left\|E_{\lambda} \phi\right\|^{2}<\infty
$$

Let $k>0$ and $\epsilon>0$, such that $\int_{k}^{+\infty} e^{2 T \lambda} d\left\|E_{\lambda} \phi\right\|^{2}<\epsilon / 8$. If $\sigma_{1}=\left(\alpha_{1}, \beta_{1}\right)$ and $\sigma_{2}=\left(\alpha_{2}, \beta_{2}\right)$. Then,

$$
\begin{aligned}
& \left\|u_{\sigma_{1}}(0)-u_{\sigma_{2}}(0)\right\|^{2} \\
& =\int_{\eta}^{+\infty}\left(\frac{1}{\beta_{1}\left(1+\left(\lambda /\left(1+\alpha_{1} \lambda\right)\right)\right)+e^{-\left(\lambda T /\left(1+\alpha_{1} \lambda\right)\right)}}-\frac{1}{\beta_{2}\left(1+\left(\lambda /\left(1+\alpha_{2} \lambda\right)\right)\right)+e^{\left.-\left(\lambda T / 1+\alpha_{2} \lambda\right)\right)}}\right)^{2} d\left\|E_{\lambda} \phi\right\|^{2} \\
& \leq 2 \int_{\eta}^{+\infty}\left[\frac{\beta_{1}\left(1+\left(\lambda /\left(1+\alpha_{2} \lambda\right)\right)\right)-\beta_{2}\left(1+\left(\lambda /\left(1+\alpha_{1} \lambda\right)\right)\right)}{\left(\beta_{1}\left(1+\left(\lambda /\left(1+\alpha_{1} \lambda\right)\right)\right)+e^{-\left(\lambda T /\left(1+\alpha_{1} \lambda\right)\right)}\right)\left(\beta_{2}\left(1+\lambda / 1+\alpha_{2} \lambda\right)+e^{-\left(\lambda T /\left(1+\alpha_{2} \lambda\right)\right)}\right)}\right]^{2} d\left\|E_{\lambda} \phi\right\|^{2} \\
& +2 \int_{\eta}^{+\infty}\left[\frac{e^{-\lambda T / 1+\alpha_{1} \lambda}-e^{-\lambda T / 1+\alpha_{2} \lambda}}{\left(\beta_{1}\left(1+\lambda /\left(1+\alpha_{1} \lambda\right)\right)+e^{-\left(\lambda T /\left(1+\alpha_{1} \lambda\right)\right)}\right)\left(\beta_{2}\left(1+\lambda /\left(1+\alpha_{2} \lambda\right)\right)+e^{-\left(\lambda T /\left(1+\alpha_{2} \lambda\right)\right)}\right)}\right]^{2} d\left\|E_{\lambda} \phi\right\|^{2} .
\end{aligned}
$$

If we put

$$
\begin{gathered}
M_{\sigma_{1}, \sigma_{2}}=\frac{\left|\beta_{1}\left(1+\left(\lambda /\left(1+\alpha_{2} \lambda\right)\right)\right)-\beta_{2}\left(1+\left(\lambda /\left(1+\alpha_{1} \lambda\right)\right)\right)\right|}{\left(\beta_{1}\left(1+\lambda /\left(1+\alpha_{1} \lambda\right)\right)+e^{-\lambda T / 1+\alpha_{1} \lambda}\right)\left(\beta_{2}\left(1+\left(\lambda /\left(1+\alpha_{2} \lambda\right)\right)\right)+e^{-\lambda T / 1+\alpha_{2} \lambda}\right)}, \\
N_{\sigma_{1}, \sigma_{2}}=\frac{\left|e^{-\left(\lambda T /\left(1+\alpha_{1} \lambda\right)\right)}-e^{-\left(\lambda T /\left(1+\alpha_{2} \lambda\right)\right)}\right|}{\left(\beta_{1}\left(1+\lambda /\left(1+\alpha_{1} \lambda\right)\right)+e^{-\left(\lambda T /\left(1+\alpha_{1} \lambda\right)\right)}\right)\left(\beta_{2}\left(1+\left(\lambda /\left(1+\alpha_{2} \lambda\right)\right)\right)+e^{-\left(\lambda T /\left(1+\alpha_{2} \lambda\right)\right)}\right)},
\end{gathered}
$$

then we have

$$
\left\|u_{\sigma_{1}}(0)-u_{\sigma_{2}}(0)\right\|^{2} \leqslant 2 \int_{\eta}^{+\infty} M_{\sigma_{1}, \sigma_{2}}^{2}(\lambda) d\left\|E_{\lambda} \phi\right\|^{2}+2 \int_{\eta}^{+\infty} N_{\sigma_{1}, \sigma_{2}}^{2}(\lambda) d\left\|E_{\lambda} \phi\right\|^{2} .
$$

Using analogous calculations as in the proof of Theorem 3.1, we obtain

$$
\begin{aligned}
\left\|u_{\sigma_{1}}(0)-u_{\sigma_{2}}(0)\right\|^{2} \leqslant & \frac{\epsilon}{2}+2 e^{2 k T}\|\phi\|_{C_{1}}^{2} \\
& \times\left[\left[\beta_{2}\left(1+\frac{k}{1+\alpha_{2} k}\right)-\beta_{1}\left(1+\frac{k}{1+\alpha_{1} k}\right)\right]^{2}+T^{2} k^{4}\left(\alpha_{1}-\alpha_{2}\right)^{2}\right] .
\end{aligned}
$$

Now, if $\sigma_{0}=(0,0)$, then by choosing $\sigma=(\alpha, \beta)$ such that

$$
|\sigma|^{2}=\alpha^{2}+\beta^{2} \leqslant \frac{1}{\|\phi\|_{C_{1}}^{2}} \frac{1}{e^{2 k T}}\left(1+\frac{1}{T^{2} k^{4}}\right) \frac{\epsilon}{8}
$$


International Journal of Differential Equations

we find that $\left\|u_{\sigma}(0)-u_{\sigma_{0}}(0)\right\|^{2}=\left\|\phi_{\sigma}-\phi_{0}\right\|^{2} \leq \epsilon$. This means that the function $F$ is continuous at $(0,0)$.

We note that we can easily show that

$$
\left\|u_{\sigma}(0)-u(0)\right\|^{2}=\left\|u_{\sigma}(T)-\phi\right\|_{C_{1}}^{2}
$$

for $\phi \in C_{1}(A)$. And by using Theorem 3.1 again, we see that

$$
\left\|u_{\sigma}(0)-u(0)\right\|^{2} \longrightarrow 0 \quad \text { as }|\sigma| \longrightarrow 0
$$

Theorem 3.4. If $\phi \in C_{\theta+1}(A)$, then one has

$$
\left\|u_{\sigma}(0)-u(0)\right\|^{2} \leqslant 2\left(\frac{4}{\alpha^{2}} C_{1}^{2}(\theta) \beta^{2 \theta}+C_{2}^{2}(\theta, T) \alpha^{2}\right)\|\phi\|_{C_{\theta+1}}^{2},
$$

for $0<\theta<1$. And

$$
\left\|u_{\sigma}(0)-u(0)\right\|^{2} \leqslant 2\left(\frac{4 \beta^{2}}{\alpha^{2}}+C_{2}^{2}(\theta, T) \alpha^{2}\right)\|\phi\|_{C_{\theta+1}}^{2}, \text { for } \theta \geqslant 1
$$

such that

$$
C_{1}(\theta)=(1-\theta)^{1-\theta} \theta^{\theta} \leq 1, \quad C_{2}(\theta, T)=\frac{1}{T \theta^{2}}
$$

Proof. Since

$$
\begin{aligned}
\left\|u_{\sigma}(0)-u(0)\right\|^{2} & =\left\|u_{\sigma}(T)-\phi\right\|_{C_{1}}^{2} \\
& =\int_{\eta}^{+\infty} P_{\sigma}^{2}(\lambda) e^{2(1+\theta) T \lambda} e^{-2 T \lambda \theta} d\left\|E_{\lambda} \phi\right\|^{2}, \\
& \leqslant 2\left(M_{\sigma, \theta, \infty}^{2}+N_{\sigma, \theta, \infty}^{2}\right)\|\phi\|_{C_{\theta+1}^{\prime}}^{2}
\end{aligned}
$$

then using Theorem 3.2, we get the required result.

Corollary 3.5. If $\phi \in C_{\theta+1}(A)$, then one has

$$
\begin{aligned}
\sup _{0 \leqslant t \leqslant T}\left\|u_{\sigma}(t)-u(t)\right\|^{2} & \leqslant\left\|u_{\sigma}(0)-u(0)\right\|^{2} \\
& \leqslant 2\left(\frac{4}{\alpha^{2}} C_{1}^{2}(\theta) \beta^{2 \theta}+C_{2}^{2}(\theta, T) \alpha^{2}\right)\|\phi\|_{C_{\theta+1}}^{2}
\end{aligned}
$$


for $0<\theta<1$. And

$$
\begin{aligned}
\sup _{0 \leqslant t \leqslant T}\left\|u_{\sigma}(t)-u(t)\right\|^{2} & \leqslant\left\|u_{\sigma}(0)-u(0)\right\|^{2} \\
& \leqslant 2\left(\frac{4 \beta^{2}}{\alpha^{2}}+C_{2}^{2}(\theta, T) \alpha^{2}\right)\|\phi\|_{C_{\theta+1}}^{2} \quad \text { for } \theta \geqslant 1 .
\end{aligned}
$$

Proof. Using Theorem 3.3, we have

$$
\sup _{0 \leqslant t \leqslant T}\left\|u_{\sigma}(t)-u(t)\right\|^{2} \leqslant\left\|u_{\sigma}(0)-u(0)\right\|^{2}
$$

And, by Theorem 3.4, we obtain the desired result.

Remark 3.6. We note that in a very recent paper by Tuan [17] a new use of a different regularization method (the truncation method) is introduced for dealing with a similar class of problems. This truncation method consists in eliminating all high frequencies from the solution of the considered ill-posed problem to get an approximate regularized solution together with some stability and error estimates that he indicates to be of Holder type. In particular, the author gives some estimates which hold at $t=0$ and so he gets the convergence of the approximate solution at $t=0$. For a significant comparison with these results obtained by this truncation regularization method, one needs the determination and selection, for each case, of a possible appropriate regularization parameter $\beta(\epsilon)$. However, the method of regularization presented in our work still gives a better approximation than many other quasireversibility and quasi-boundary type methods, for example, [1, 6, 7, 19-21].

Conclusion 1. (1) Note that, in this work, the error factor $\varepsilon(\sigma)$ introduced by small changes in the final value is of order $1 /\left(\beta(1+(\eta /(1+\alpha \eta)))+e^{-T / \alpha}\right)$ and in a recent work [21], by the same authors, the error factor given was of order $1 /\left(\beta \eta /(1+\alpha \eta)+e^{-T / \alpha}\right) \geq 1 /(\beta(1+(\eta /(1+$ $\alpha \eta))+e^{-T / \alpha}$.

(2) We also note that the error factor $e(\beta)$ given in [7] (resp. $e(\alpha)$ in [1]) is of order $1 / \beta$ (resp., $e^{T / \alpha}$ ) and so $1 /\left(\beta \eta /(1+\alpha \eta)+e^{-T / \alpha}\right) \leq 1 / \beta$ and $1 /\left(\beta \eta /(1+\alpha \eta)+e^{-T / \alpha}\right) \leq e^{T / \alpha}$.

(3) Also the error factor $e(\sigma)$ given in Boussetila and Rebbani [19] is of order $1 /(\beta+$ $\left.e^{-T / \alpha}\right)$ and since $1 /\left(\beta(1+(\eta /(1+\alpha \eta)))+e^{-T / \alpha}\right) \leq 1 /\left(\beta+e^{-T / \alpha}\right)$, for $0<\alpha \leq 1-1 / \eta, \eta>1$ and so for all operators $A(A \geq \eta>1)$, considered above, our method of approximation gives a better approximation than the methods given by Boussetila and Rebbani in [19] and other authors, for example, $[1,7]$.

\section{References}

[1] R. Lattès and J.-L. Lions, Méthode de Quasi-Réversibilité et Applications, Travaux et Recherches Mathématiques, no. 15, Dunod, Paris, France, 1967.

[2] M. M. Lavrentiev, Some Improperly Posed Problems of Mathematical Physics, vol. 11 of Springer Tracts in Natural Philosophy, Springer, Berlin, Germany, 1967.

[3] K. Miller, "Stabilized quasi-reversibility and other nearly-best-possible methods for non- well-posed problems," in Symposium on Non-Well Posed Problems and Logarithmic Convexity (Heriot-Watt University, Edinburgh, Scotland, 1972), vol. 316 of Lecture Notes in Mathematics, pp. 161-176, Springer, Berlin, Germany, 1973. 
[4] L. E. Payne, "Some general remarks on improperly posed problems for partial differential equations," in Symposium on Non-Well Posed Problems and Logarithmic Convexity (Heriot-Watt University, Edinburgh, Scotland, 1972), vol. 316 of Lecture Notes in Mathematics, pp. 1-30, Springer, Berlin, Germany, 1973.

[5] R. E. Showalter, "The final value problem for evolution equations," Journal of Mathematical Analysis and Applications, vol. 47, no. 3, pp. 563-572, 1974.

[6] M. Ababna, "Regularization by nonlocal conditions of the problem of the control of the initial condition for evolution operator-differential equations," Vestnik Belorusskogo Gosudarstvennogo Universiteta. Seriya 1. Fizika, Matematika, Informatika, vol. 81, no. 2, pp. 60-63, 1998 (Russian).

[7] G. W. Clark and S. F. Oppenheimer, "Quasi-reversibility methods for non-well posed problems," Electronic Journal of Differential Equations, no. 8, pp. 1-9, 1994.

[8] R. E. Showalter, "Cauchy problem for hyper-parabolic partial differential equations," North-Holland Mathematics Studies, vol. 110, no. C, pp. 421-425, 1985.

[9] V. K. Ivanov, I. V. Mel'nikova, and A. I. Filinkov, Differentsialno-Operatornye Uravneniya i Nekorrektnye Zadachi, Fizmatlit "Nauka", Moscow, Russia, 1995.

[10] I. V. Mel'nikova, "Regularization of ill-posed differential problems," Sibirskii Matematicheskii Zhurnal, vol. 33, no. 2, pp. 125-134, 1992 (Russian).

[11] I. V. Mel'nikova, "Regularization of ill-posed differential problems," Siberian Mathematical Journal, vol. 33, no. 2, pp. 289-298, 1992.

[12] K. A. Ames and L. E. Payne, "Asymptotic behavior for two regularizations of the Cauchy problem for the backward heat equation," Mathematical Models \& Methods in Applied Sciences, vol. 8, no. 1, pp. 187-202, 1998.

[13] K. A. Ames, L. E. Payne, and P. W. Schaefer, "Energy and pointwise bounds in some non-standard parabolic problems," Proceedings of the Royal Society of Edinburgh. Section A. Mathematics, vol. 134, no. 1, pp. 1-9, 2004.

[14] M. Denche and S. Djezzar, "A modified quasi-boundary value method for a class of abstract parabolic ill-posed problems," Boundary Value Problems, vol. 2006, Article ID 37524, 8 pages, 2006.

[15] S. Djezzar, "Regularization method for an abstract backward Cauchy problem," in Proceedings of the 3rd International Conference on Mathematical Sciences (ICM '08), vol. 3, pp. 1116-1125, March 2008.

[16] B. M. Campbell Hetrick and R. J. Hughes, "Continuous dependence results for inhomogeneous illposed problems in Banach space," Journal of Mathematical Analysis and Applications, vol. 331, no. 1, pp. 342-357, 2007.

[17] N. H. Tuan, "Regularization for a class of backward parabolic problems," Bulletin of Mathematical Analysis and Applications, vol. 2, no. 2, pp. 18-26, 2010.

[18] N. H. Tuan, D. D. Trong, and P. H. Quan, “On a backward Cauchy problem associated with continuous spectrum operator," Nonlinear Analysis, vol. 73, no. 7, pp. 1966-1972, 2010.

[19] N. Boussetila and F. Rebbani, "Optimal regularization method for ill-posed Cauchy problems," Electronic Journal of Differential Equations, no. 147, pp. 1-15, 2006.

[20] M. Denche and K. Bessila, "A modified quasi-boundary value method for ill-posed problems," Journal of Mathematical Analysis and Applications, vol. 301, no. 2, pp. 419-426, 2005.

[21] S. Djezzar and N. Teniou, "Modified regularization method for backward Cauchy problems," in Proceedings of the 3rd Conference on Mathematical Sciences (CMS '11), pp. 1512-1519, Zarqa, Jordan, April 2011.

[22] A. Pazy, "Semi-groups of linear operators and applications to partial differential equations," in Applied Mathematical Sciences, vol. 44, Springer, New York, NY, USA, 1983. 


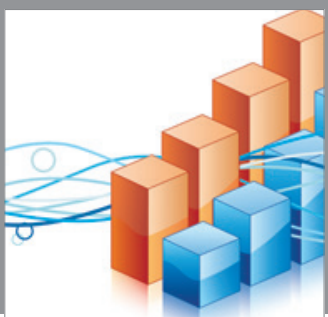

Advances in

Operations Research

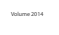

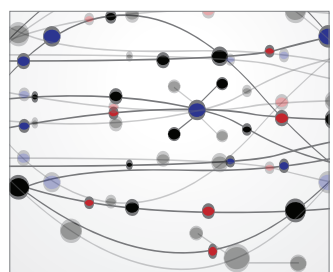

\section{The Scientific} World Journal
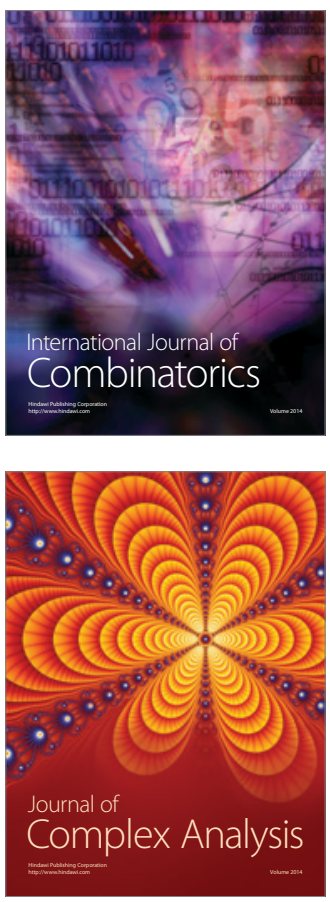

International Journal of

Mathematics and

Mathematical

Sciences
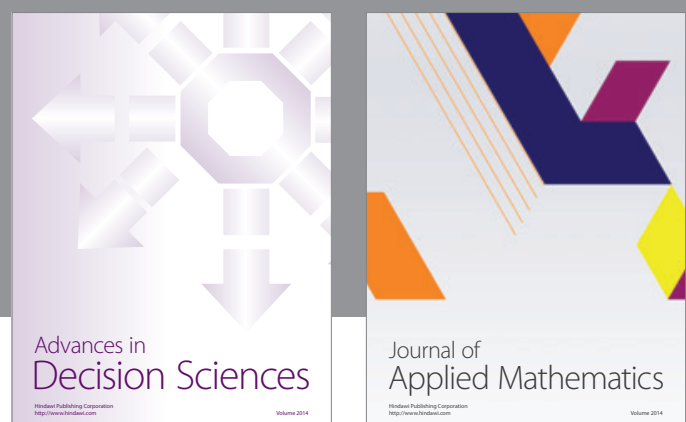

Journal of

Applied Mathematics
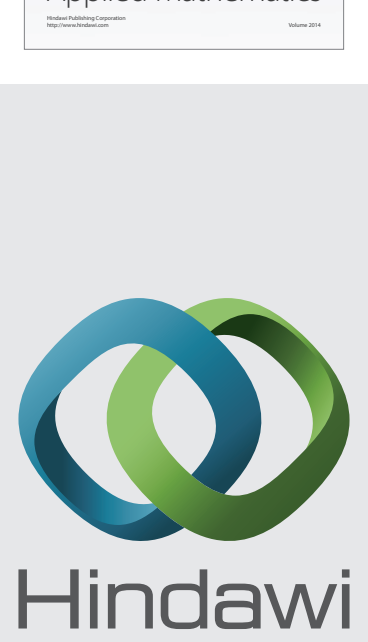

Submit your manuscripts at http://www.hindawi.com
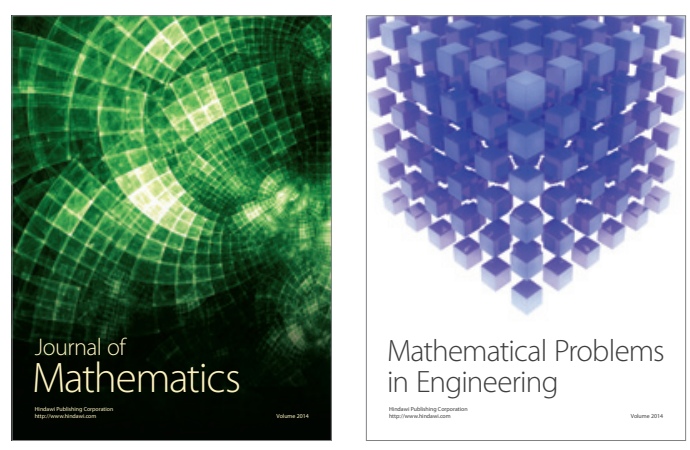

Mathematical Problems in Engineering
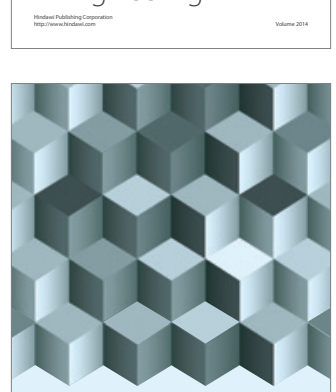

Journal of

Function Spaces
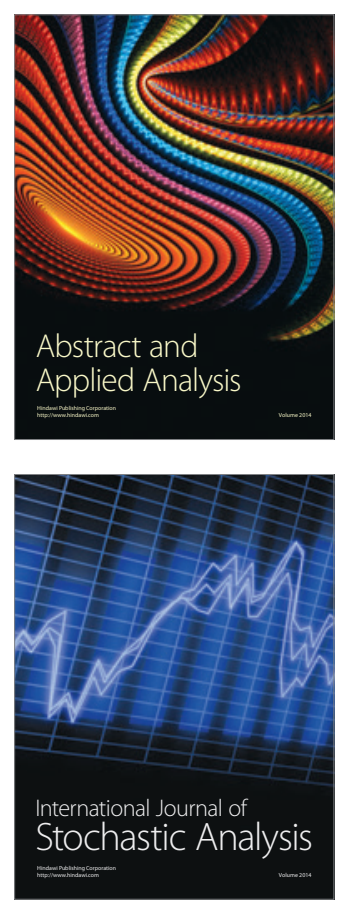

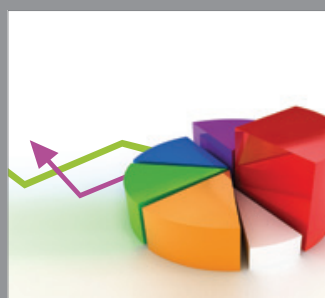

ournal of

Probability and Statistics

Promensencen
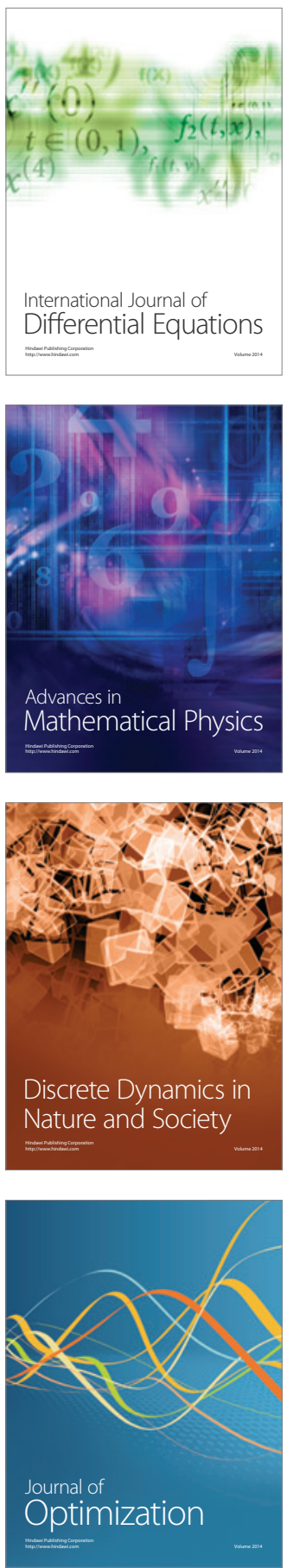